\title{
Barriers to Treatment in Patients With Locally Advanced Breast Cancer
}

\author{
Laura Bourdeanu, $\mathbf{P h D}^{\mathrm{a}}$, Thehang Luu, $\mathbf{M D}^{\mathrm{b}}$, Norma Baker, $\mathbf{R N}^{\mathrm{b}}$, Suzanne Swain-Cabriales, \\ RN, MSN ${ }^{\mathrm{c}}$, Cathie T. Chung, MD $^{\mathrm{b}}$, Joanne Mortimer, MD $^{\mathrm{b}}$, Arti Hurria, $\mathbf{M D}^{\mathrm{b}}$, Sandra Helton, \\ $\mathbf{R N}^{\mathrm{b}}$, David Smith, $\mathbf{P h D}^{\mathrm{d}}$, Betty Ferrell, $\mathbf{P h D}^{\mathrm{e}}$, Gloria Juarez, $\mathbf{P h D}^{\mathrm{e}}$, and George Somlo, $\mathbf{M D}^{\mathrm{b}}$ \\ aDepartment of Nursing, Sage College, Troy, New York \\ bepartment of Medical Oncology and Therapeutics Research, City of Hope Comprehensive \\ Cancer Center, Duarte, California \\ 'Division of Medical Oncology, Cedars Sinai-Samuel Oschin Cancer Center, Los Angeles, \\ California \\ ${ }^{\mathrm{d}}$ Division of Biostatistics, Department of Information Sciences, City of Hope Comprehensive \\ Cancer Center, Duarte, California \\ eDivision of Nursing Research and Education, Department of Population Science, City of Hope \\ Comprehensive Cancer Center, Duarte, California
}

\begin{abstract}
Delays between presentation and treatment could have a significant effect on breast cancer mortality. The authors hypothesized that patient, physician, and system barriers are all responsible for treatment delays. Therefore, a study was conducted to define prevalent barriers to treatment from the patient's perspective. A modified 43-item Likert-scale questionnaire was administered to patients with clinical stage III locally advanced breast cancer (LABC) who had experienced a delay in treatment of 3 months or more. Between October 2008 and January 2010, 153 patients presented with LABC; 43 patients $(28.1 \%)$ met eligibility, and 40 completed the questionnaire. Among the patient barriers reported, $38 \%$ of patients delayed care for fear of losing their breast and $47 \%$ awaited previously scheduled routine appointments instead of seeking care. Among the physician barriers reported, $20 \%$ of physicians of initial contact did not believe the breast lump/ symptom was related to cancer and $15 \%$ did not believe it needed a biopsy. Among the system barriers reported, the most prevalent were delays in performing diagnostic tests and obtaining insurance authorization for tests, treatment, or physician visits. Substantial delays were seen in $28.1 \%$ of patients from presentation to when they sought therapy at City of Hope Comprehensive Cancer Center. The high prevalence of patient barriers versus physician/system barriers suggests that increased educational efforts for patients and health care professionals are needed.
\end{abstract}

Correspondence: George Somlo, MD, Department of Medical Oncology and Therapeutics Research, City of Hope Comprehensive Cancer Center, Duarte, CA 91010. gsomlo@coh.org.

A report of this work was presented at the 46th Annual Meeting of the American Society of Clinical Oncology, June 4-8, 2010, Chicago, Illinois Abstract 6040.

The authors have disclosed that they have no financial interests, arrangements, affiliations, or commercial interests with the manufacturers of any products discussed in this article or their competitors. 
In the United States, breast cancer will affect approximately 1 in 8 women, resulting in an estimated 132,340 new cases of invasive breast cancer and 39,620 deaths in 2013. ${ }^{1}$ Despite efforts to improve early detection, too many patients still present for treatment after extended delays from the initial onset of symptoms. ${ }^{2}$ According to one definition, delay is considered a period of at least 3 months from symptom appearance to seeking medical care. ${ }^{3}$ Approximately one-fourth of women with breast cancer symptoms have at least a 3-month delay in care. ${ }^{1}$ Delay in care is likely to affect prognosis and survival. ${ }^{4}$ As an example, increased tumor size is associated with a greater likelihood of regional and distant metastasis, and long-term survival is worse with each $5 \mathrm{~mm}$ of growth. ${ }^{5-8}$

In an effort to reduce cancer disparities, interventions to improve breast cancer screening have been successfully implemented. ${ }^{9,10}$ However, the authors observed that a disproportionately high number of patients continued to present with locally advanced breast cancer at City of Hope National Medical Center, an NCIdesignated Comprehensive Cancer Center (COHCC) located in Southern California, where access to health care resources is readily available. The authors hypothesized that 3 major primary roots for delays originate primarily from the patient, physician, or health care system. The study intended to determine the perceived barriers to treatment among a cohort of women presenting at $\mathrm{COHCC}$ with locally advanced or inflammatory breast cancer who had experienced a 3-month or greater delay in initiation of oncologic treatment from the time of the initial event that led them to seek treatment.

\section{Methods}

\section{Study Population}

This pilot study enrolled patients from an ethnically, socioeconomically diverse population drawn primarily from a 50-mile radius area encompassing greater Los Angeles, San Bernardino County, and surrounding areas. The sample size was based on COHCC's accrual patterns and was specified in advance, with primary consideration for the time allotted for the study. All patients signed an informed, voluntary, COHCC Internal Review Boardapproved consent form. Eligible patients were recruited between October 2008 and January 2010 who fulfilled the following criteria: 1 ) were diagnosed with histologically proven clinical stage III and/or inflammatory breast cancer (defined clinically as having one-third or more of the breast surface area involved with inflammatory changes); 2) male or female; 3) had no history of prior malignancy; 4) were 18 years of age or older; 5) experienced a delay of 3 months or more in initiation of oncologic treatment from the time of the symptom occurrence/reason for seeking treatment; and 6) were proficient in English. During the accrual period, 153 patients presented with locally advanced stage III (LABC) or inflammatory breast cancer. Forty-three patients $(28.1 \%)$ were eligible and, of sequentially screened patients, 40 (93.0\%) agreed to participate. Participants were approached either at the first appointment with the oncologist or at the first chemotherapy treatment.

\section{Statistical Considerations}

This study used a prospective descriptive study design. Nichols et al's ${ }^{11} 3$-phase model was used to better categorize the delay: 1) the interval between first noticing a symptom and first 
consulting a doctor (ie, patient delay); 2) the interval between first consultation with a doctor and referral (ie, practitioner delay); and 3) the time between referral and diagnosis (ie, system delay).

\section{Assessment Tool}

Patients were asked to complete a modified 43-item Likert-Scale Barriers Scale for Oncologic Treatment Questionnaire developed to explore perceived barriers. The questionnaire was developed based on previous studies with qualitative data from patient and focus group interviews. ${ }^{12-18}$ Responses were rated on a 5-point scale of "strongly agree" through "strongly disagree," relative to the barriers presented (Table 1). The dates associated with the symptom occurrence/reason for seeking treatment were obtained from the participants. Dates of the biopsies, followup appointments, imaging studies, referrals, and demographic data were obtained from the participants' medical records, allowing the investigators to determine where the delays occurred and to assess which kinds of delays were most frequent and of longest duration.

The responses to the questionnaire were tabulated using frequency tables and constructed histograms for graphic representation. The analyses were limited to descriptive statistics. This pilot cohort had a lack of sufficient inferential power for correlating demographic and clinical factors with subject-reported barriers, particularly when subjects identified multiple types of barriers simultaneously.

\section{Results}

\section{Patients}

Forty eligible and evaluable patients (median age, 49 years; range, 30-78 years) were consecutively enrolled over 12 months and completed the questionnaire. Of these, $32.5 \%$ were Caucasian, $47.5 \%$ Hispanic, $12.5 \%$ African American, and 7.5\% Asian. This is somewhat different from the ethnic distribution of all patients with LABC seen at COHCC during the enrollment period, which included 59.5\% Caucasian, 28.8\% Hispanic, 7.8\% African American, and $10.5 \%$ Asian. The characteristics of the study population are shown in Table 2. Patients experienced a range of 16- to 77-week delays (median, 24 weeks) to initiating chemotherapy treatment. For $75 \%$ of patients, the diagnosis of breast cancer was made at an outside institution. Of the patients diagnosed at an outside institution, $6.7 \%$ had Medicare, $46.0 \%$ had state medical insurance, $40.0 \%$ had private insurance, and only $6.7 \%$ had no insurance. The insurance distribution of the aforementioned participants is different from that of the patients diagnosed at COHCC, among whom 30\% had Medicare, 20\% state medical insurance, $40 \%$ private insurance, and $10 \%$ no insurance.

\section{Barriers to Treatment as Reported by Patients}

Patient Barriers-Patients reported multiple barriers in this category; however, the most commonly reported patient barrier, based on participants reporting "strongly agree" or "agree," was "patient waited for the previously scheduled standard appointment to get the results instead of requesting an earlier appointment" (47.5\%). Approximately $38 \%$ of patients did not seek medical care because they were concerned about losing their breast. 
Other patients delayed care because of the perception that their breast symptoms were from infection or muscle strain, or related to their menstrual cycle $(25.0 \%)$; that the lumps would go away $(28.5 \%)$; that the lumps were not important or urgent $(20.0 \%)$; or they did not want to think about the breast changes or lumps (20.0\%). Roughly one-fourth of the patients $(22.5 \%)$ were worried that they could not afford the copayment. Other barriers, such as access to transportation, lack of insurance, inconvenient physician office hours, child care problems, lack of family support, other medical and emotional problems, and inability to take time off from work, continued to be present but were not as frequently reported, with each of these barriers being reported less than $20 \%$ of the time.

Physician Barriers-The most commonly reported physician barriers per patient reports were "the physician of initial contact did not believe their breast lump/symptom was related to cancer" $(20 \%)$ or did not think a biopsy was necessary $(15 \%)$. Ten percent of the physicians did not refer the patient for further evaluation.

System Barriers-The most prevalent system barriers were "delay in scheduling diagnostic test" $(40.0 \%)$ and "delays from insurance company in authorizing tests, treatment, or doctor visits" $(30.0 \%)$. Less frequent were delays in referral to the oncologist $(21.1 \%)$ and delays in getting the test results $(17.5 \%)$. Only $12.9 \%$ of the patients reported having a negative initial biopsy.

\section{Time Lapsed Between Occurrence of Symptoms and Stages of Evaluation-}

Table 3 shows the ranges and medians for the time between symptom occurrence/reason for seeking treatment and appointments related to seeking treatment, and between appointments marking various stages of evaluation. The range for overall time elapsed between symptom occurrence and beginning of chemotherapy was 16 to 77 weeks (median, 24 weeks). The time between the first symptom occurrence/reason for seeking treatment and the first appointment ranged from 0 to 72 weeks (median, 12 weeks), with 0 indicating that the patient had a diagnostic or physician appointment the same week the symptoms occurred. Of the 40 participants, 22 had an appointment within 3 months or less. The time to achieving histologic diagnosis was 3 to 72 weeks (median, 15 weeks), with 15 patients having a biopsy within 3 months or less. Most of the patients had almost every one of the combinations of delays delineated in Table 3.

\section{Discussion}

This study investigated the variables associated with a 3-month or greater delay leading to the eventual treatment of LABC from the time of onset of breast cancer symptoms based on the responses to the Barriers Scale for Oncologic Treatment Questionnaire. Unexpectedly, $55 \%$ of the delays observed were patient-related only, with an additional $27.5 \%$ of the delays being a combination of patient and system or physician delays. The most frequent causes included patients waiting for a previously scheduled standard/annual appointment to be evaluated instead of requesting an earlier appointment to address their concerns, or patients' concern about losing their breast. These findings contrast strikingly with previous studies in which only $9 \%$ to $45 \%$ of patients were found to have patient-related delays, with the most cited reason why women waited to seek medical attention being fear of finding 
cancer. Although these fears continue to cause major barriers for women $(17.5 \%$ in this study), the authors can speculate that their patient population experienced fewer system barriers than seen in previous studies.

Physician delay has been reported for $6 \%$ to $18 \%$ and system delay for up to $61 \%$ of patients with breast cancer who experienced therapeutic delay. ${ }^{12,19}$ In this study, only $15 \%$ of the participants experienced physician and/or system delays, with an additional $27.5 \%$ experiencing a combination of patient and system or physician delays. The most commonly reported physician barrier was the physician of initial contact not believing that the breast lump/symptom was related to cancer, with $15 \%$ of physicians making a judgment that a biopsy was not necessary because of the unlikely possibility of finding cancer. Delays in scheduling diagnostic tests and insurance continued to be system barriers that lengthened the delay in oncologic treatment.

A limitation of this study is the difficulty establishing the exact date of symptom onset, because the authors relied on the date of symptom occurrence/reason for seeking treatment as recalled by the patients at the time they entered the study. Because of the singleinstitutional nature of the study, another limitation is the sample size: it is too small to determine whether the delays to seeking treatment differed from those in previous studies or among ethnicities evaluated. Despite the small sample size, however, the findings clearly show the existence of barriers to seeking and receiving oncologic treatment among women with access to specialists.

\section{Conclusions}

These findings suggest that delay by the patient in seeking medical care immediately after the onset of breast symptoms is a major barrier to oncologic treatment, as evidenced by the length of patient delays being longer than those caused by the provider or system. These results indicate that the system and physician barriers are not as prevalent as reported in other studies. Hence, although it is important to continue to address the physician and system barriers that remain, a need exists to direct greater resources toward educating patients and the population about the importance of assertively seeking early medical assessment, and to share information about the significant advances yielding improved survival and quality of life outcomes, so that patients, family, and society can overcome the fear of treatment.

Larger studies clearly are essential to confirm the findings of this pilot study and gain further insight into the barriers and disparities to oncologic treatment. This understanding will, in turn, allow the development of interventions to decrease delays in treatment, which should reduce breast cancer mortality.

\section{Acknowledgments}

Editorial assistance in the preparation of the manuscript was provided by Dr. Carol Wuenschell, a scientific writer employed by City of Hope. 
Research reported in this publication was supported by National Cancer Institute of the National Institutes of Health under grant number P30CA033572. The content is solely the responsibility of the authors and does not necessarily represent the official views of the National Institutes of Health.

\section{References}

1. Siegel R, Naishadham D, Jemal A. Cancer statistics, 2013. CA Cancer J Clin. 2013; 63:11-30. [PubMed: 23335087]

2. Wingo PA, Cardinez CJ, Landis SH, et al. Long-term trends in cancer mortality in the United States, 1930-1998. Cancer. 2003; 97:3133-3275. [PubMed: 12784323]

3. Pack GT, Gallo JS. Culpability for delay in the treatment of cancer. Am J Cancer. 1938; 33:443462.

4. Bish A, Ramirez A, Burgess C, et al. Understanding why women delay in seeking help for breast cancer symptoms. J Psychosom Res. 2005; 58:321-326. [PubMed: 15992567]

5. Arndt V, Sturmer T, Stegmaier C, et al. Patient delay and stage of diagnosis among breast cancer patients in Germany-a population based study. Br J Cancer. 2002; 86:1034-1040. [PubMed: 11953844]

6. Burgess CC, Potts HW, Hamed H, et al. Why do older women delay presentation with breast cancer symptoms? Psychooncology. 2006; 15:962-968. [PubMed: 16511900]

7. Montazeri A, Ebrahimi M, Mehrdad N, et al. Delayed presentation in breast cancer: a study in Iranian women. BMC Womens Health. 2003; 3:4. [PubMed: 12846932]

8. Olivotto IA, Gomi A, Bancej C, et al. Influence of delay to diagnosis on prognostic indicators of screen-detected breast carcinoma. Cancer. 2002; 94:2143-2150. [PubMed: 12001110]

9. Legler J, Meissner HI, Coyne C, et al. The effectiveness of interventions to promote mammography among women with historically lower rates of screening. Cancer Epidemiol Biomarkers Prev. 2002; 11:59-71. [PubMed: 11815402]

10. Wells KJ, Battaglia TA, Dudley DJ, et al. Patient navigation: state of the art or is it science? Cancer. 2008; 113:1999-2010. [PubMed: 18780320]

11. Nichols S, Waters WE, Fraser JD, et al. Delay in the presentation of breast symptoms for consultant investigation. Community Med. 1981; 3:217-225. [PubMed: 7273693]

12. Goodson WH III, Moore DH II. Causes of physician delay in the diagnosis of breast cancer. Arch Intern Med. 2002; 162:1343-1348. [PubMed: 12076232]

13. Chang SW, Kerlikowske K, Napoles-Springer A, et al. Racial differences in timeliness of followup after abnormal screening mammography. Cancer. 1996; 78:1395-1402. [PubMed: 8839544]

14. Elwood JM, Moorehead WP. Delay in diagnosis and long-term survival in breast cancer. Br Med J. 1980; 280:1291-1294. [PubMed: 7388514]

15. Facione NC. Delay versus help seeking for breast cancer symptoms: a critical review of the literature on patient and provider delay. Soc Sci Med. 1993; 36:1521-1534. [PubMed: 8327915]

16. Facione NC, Dodd MJ, Holzemer W, et al. Helpseeking for selfdiscovered breast symptoms. Implications for early detection. Cancer Pract. 1997; 5:220-227. [PubMed: 9250078]

17. Ramirez AJ, Westcombe AM, Burgess CC, et al. Factors predicting delayed presentation of symptomatic breast cancer: a systematic review. Lancet. 1999; 353:1127-1131. [PubMed: 10209975]

18. Nichols S, Waters WE, Fraser JD, et al. Delay in the presentation of breast symptoms for consultant investigation. Community Med. 1981; 3:217-225. [PubMed: 7273693]

19. Ashing-Giwa KT, Gonzalez P, Lim JW, et al. Diagnostic and therapeutic delays among a multiethnic sample of breast and cervical cancer survivors. Cancer. 2010; 116:3195-3204. [PubMed: 20564623] 


\section{Table 1}

\section{Delineation of Reported Barriers}

\section{Physician-Related Barriers}

The mammogram was negative so I thought everything was fine.

The physician did not think the lump was cancer.

The physician did not think it was necessary to do a biopsy.

The physician did not refer me for further evaluation.

There were delays in the referral to the oncologist.

The biopsy was negative so I thought everything was fine.

\section{System-Related Barriers}

I had difficulty with the insurance company approving the mammogram.

There were delays in scheduling diagnostic tests.

There were delays in getting test results.

There were delays from the insurance company in authorizing tests, treatment, or doctor visits.

\section{Patient-Related Barriers}

I worried that mammograms cost too much and I did not have insurance.

I was too busy with work or school to get a mammogram or see a doctor.

I had difficulty taking time off from work.

I had difficulty finding transportation.

I just did not want to get any further tests.

I worried about the possibility of losing my breast.

I worried that I could not afford the copayment.

I thought I would have difficulty finding child care.

I thought I was too old to need a mammogram.

I thought I was too young to need a mammogram.

I did not want to think about the breast changes/lump.

I did not think the breast changes/lump was important or urgent.

I thought the changes/lump would go away.

I thought the lump was an infection, a muscle strain, or related to my period.

I thought my significant other or family would not be supportive of future treatments.

I did not make it to the follow-up appointments after I first sought medical attention.

I waited for the usual or scheduled return visit to get my results.

I had other more important health problems that prevented me from getting a mammogram.

I had emotional problems that prevented me from getting a mammogram.

I had sick family members to take care of so I couldn't attend to my breast problem.

My family would have made fun of me if I had a mammogram.

I could not remember to schedule a mammogram.

I thought having a mammogram would expose me to unnecessary radiation.

I was afraid that having a mammogram would be too painful.

I was afraid to have a mammogram because I thought having it would take too much time.

I did not know how to go about getting a mammogram.

I thought a woman doesn't need a mammogram unless she has symptoms. 
I was afraid to have a mammogram because I did not understand what would be done.

I was afraid to have a mammogram after finding the breast changes/lump because I thought it might mean something was wrong. 


\section{Table 2}

\section{Population Characteristics}

\begin{tabular}{|c|c|c|}
\hline Category & $\mathbf{N}$ & $\%$ \\
\hline All patients & 40 & \\
\hline \multicolumn{3}{|l|}{ Age (y) } \\
\hline 50 & 21 & 52.5 \\
\hline $51-60$ & 10 & 25.0 \\
\hline$>60$ & 9 & 22.5 \\
\hline \multicolumn{3}{|l|}{ Ethnicity } \\
\hline Caucasian & 13 & 32.5 \\
\hline African American & 5 & 12.5 \\
\hline Asian & 3 & 7.5 \\
\hline Hispanic & 19 & 47.5 \\
\hline \multicolumn{3}{|l|}{ Education } \\
\hline High school & 17 & 42.5 \\
\hline > High school & 23 & 57.5 \\
\hline \multicolumn{3}{|l|}{ Marital status } \\
\hline Married & 16 & 40.0 \\
\hline Single & 13 & 32.5 \\
\hline Divorced & 6 & 15.0 \\
\hline Widowed & 5 & 12.5 \\
\hline \multicolumn{3}{|l|}{ Occupation } \\
\hline Employed & 26 & 65.0 \\
\hline Housewife & 10 & 25.0 \\
\hline Disabled & 4 & 10.0 \\
\hline \multicolumn{3}{|l|}{ Insurance } \\
\hline Private & 16 & 40.0 \\
\hline Medicare & 5 & 12.5 \\
\hline Medicaid & 16 & 40.0 \\
\hline None & 3 & 7.5 \\
\hline \multicolumn{3}{|c|}{ Nature of symptoms/reasons leading to diagnosis } \\
\hline Rash & 3 & 7.5 \\
\hline Lump & 30 & 75.0 \\
\hline Pain & 4 & 10.0 \\
\hline Abnormal mammogram & 3 & 7.5 \\
\hline
\end{tabular}




\section{Table 3}

Time Lapsed Between Stages of Evaluation Barriers

\begin{tabular}{|ll|}
\hline Interval End Points & Time Lapsed (wk) \\
\hline Symptoms to first appointment & $0^{a}-72$ (median, 12) \\
Symptoms to mammogram & $0^{a}-72$ (median, 12) \\
Symptoms to pathology & $3-72$ (median, 15) \\
First appointment to pathology & $3-72$ (median, 15) \\
Surgeon to oncologist & $0^{a}-21$ (median, 2) \\
Pathology report to oncologist & $0^{a}-23$ (median, 5) \\
Oncologist to chemotherapy & $0^{a}-6$ (median, 3) \\
Symptoms to oncology appointment & $13-74$ (median, 21) \\
Symptoms to chemotherapy & $16-77$ (median, 24) \\
\hline
\end{tabular}

${ }^{a}$ Signifies that the patient saw physician the same week they noted the breast symptoms/reasons leading to diagnosis. 